\title{
Calorimeters in the Very Forward Region of ILC
}

\author{
Jinlong Zhang \\ University of Colorado
}

XII International Conference on

Calorimetry in High Energy Physics

June 9, 2006

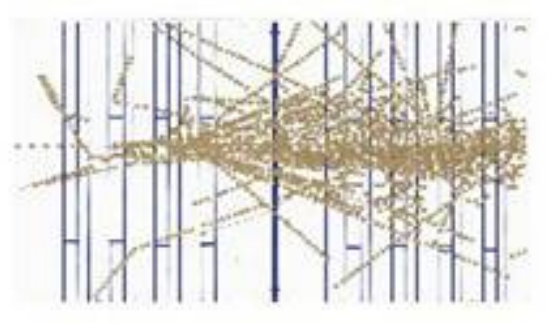




\section{FCAL Collaboration}

National Scientific and Educational Center of Particle and High Energy Physics, Belarus State University

Institute of Physics of the Academy of Sciences of the Czech Republic

\section{DESY Zeuthen}

School of Physics and Astronomy,

Tel Aviv University

Faculty of Physics and Applied Computer Science, AGH University of Science and Technology

Institute of Nuclear Physics of the Polish Academy of Sciences

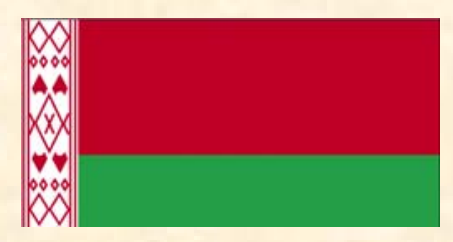

\author{
JINR Dubna \\ Institute of High Energy Physics \\ Protvino
}

"VINCA" Institute of Nuclear
Science

Royal Holloway University of

London

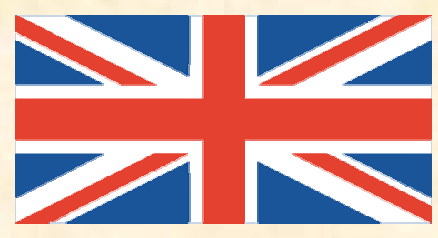

University of Colorado at Boulder
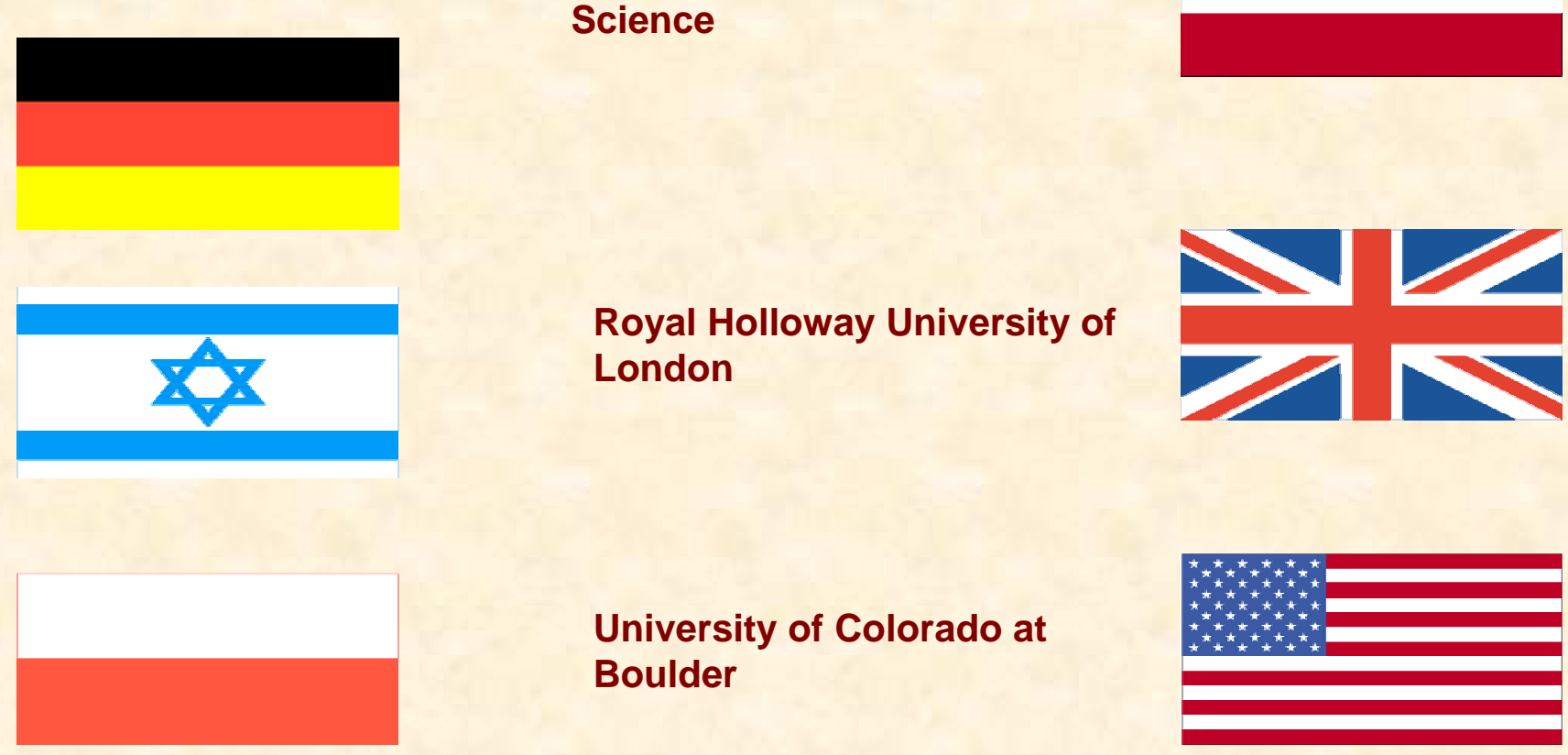

CALOR 2006 


\section{Very Forward Region}

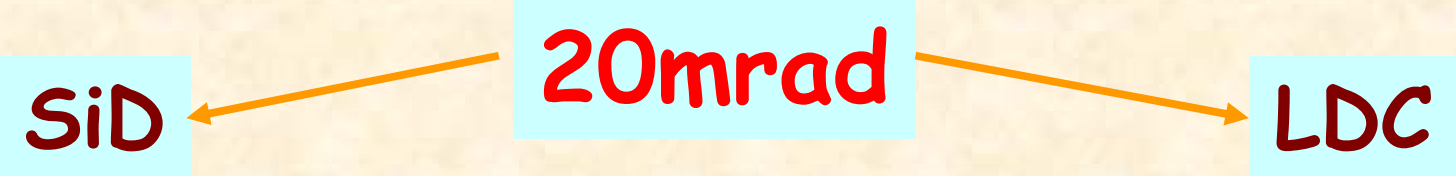

Si D Forward Masking, Calorimetry \& Tracking 2005-09-15 $20 \mathrm{mrad}, L^{*}=3.51 \mathrm{~m}$
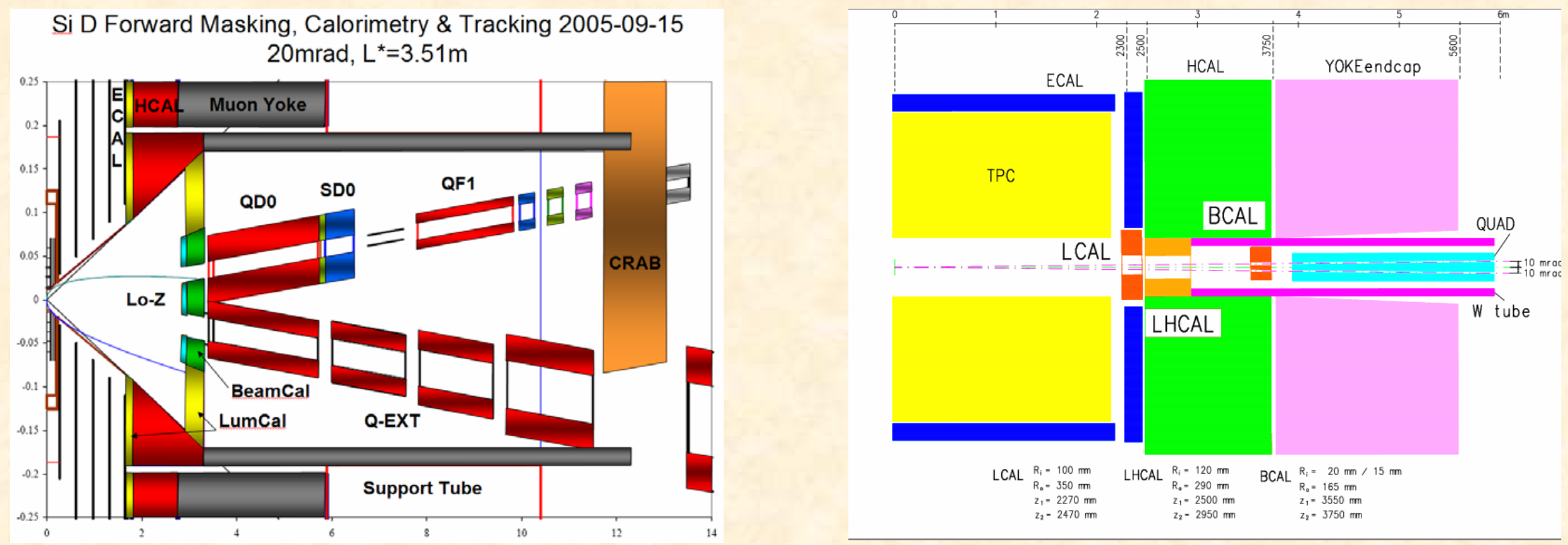

$\begin{array}{lll}5<\theta<25 \mathrm{mrad} & \text { BeamCal } & 5<\theta<45 \mathrm{mrad} \\ 25<\theta<113 \mathrm{mrad} & \text { LumiCal } & 44<\theta<155 \mathrm{mrad}\end{array}$




\section{LumiCal}

Measure the luminosity precisely

$\frac{\Delta L}{L} \cong 10^{-4}$

Extend the detector coverage

Silicon-tungsten sandwich geometry

\begin{tabular}{|l|c|c|}
\hline & $2 \mathrm{mrad}$ & $20 \mathrm{mrad}$ \\
\hline Absorber thickness $(\mathrm{mm})$ & $\sim 3.5$ & $\sim 3.5$ \\
\hline Sensor thickness $(\mathrm{mm})$ & $\sim 0.5$ & $\sim 0.5$ \\
\hline $\mathrm{X} / \mathrm{Y}$ position $(\mathrm{mm})$ & $0 / 0$ & $+23.70 / 0$ \\
\hline $\mathrm{Z}_{\min }-\mathrm{Z}_{\max }(\mathrm{mm})$ & $\pm(2270-2470)$ & $\pm(2270-2470)$ \\
\hline Tilt angle $(\mathrm{mrad})$ & 0 & 10 \\
\hline $\mathrm{R}_{\min }-\mathrm{R}_{\max }(\mathrm{mm})$ & $60-350$ & $100-350$ \\
\hline Number of layers & 30 & 30 \\
\hline
\end{tabular}




\section{Two Designs}

\section{strip}

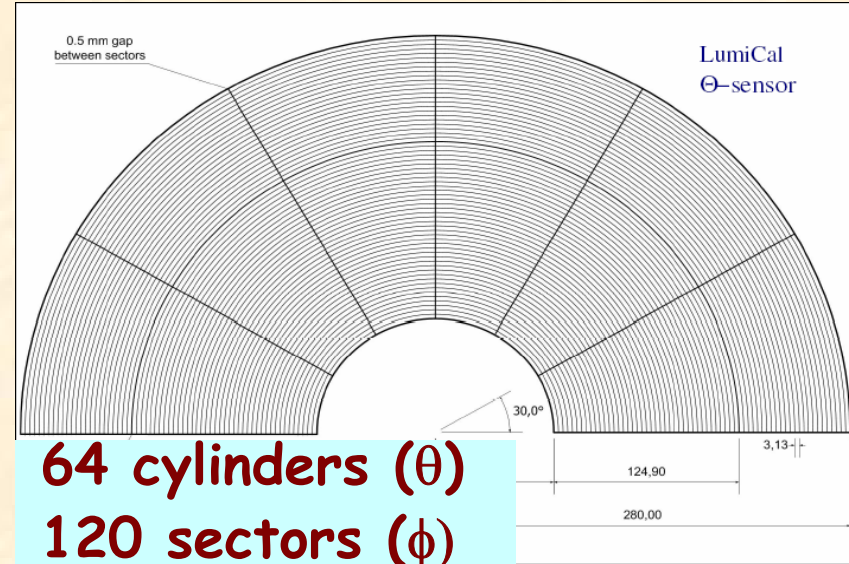

30 rings $(Z)$

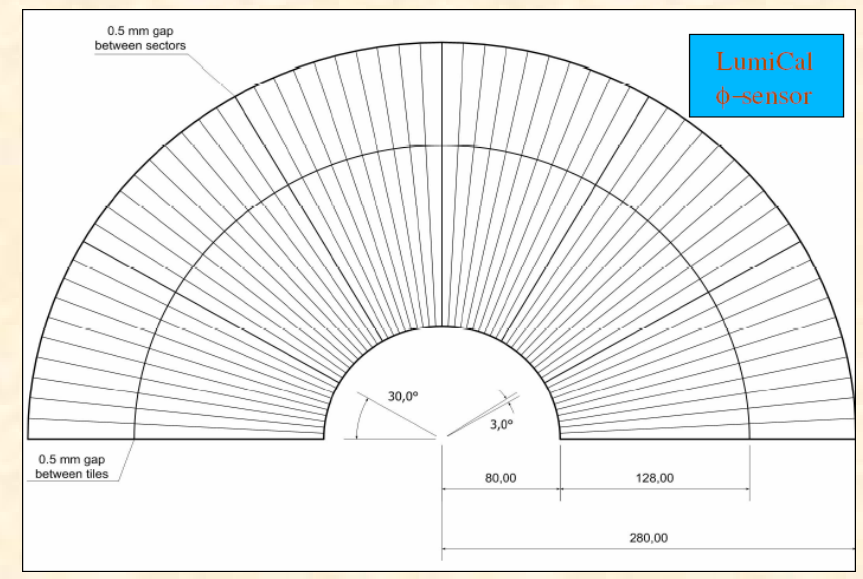

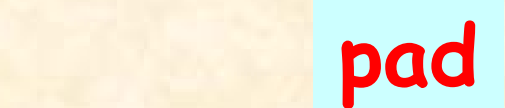

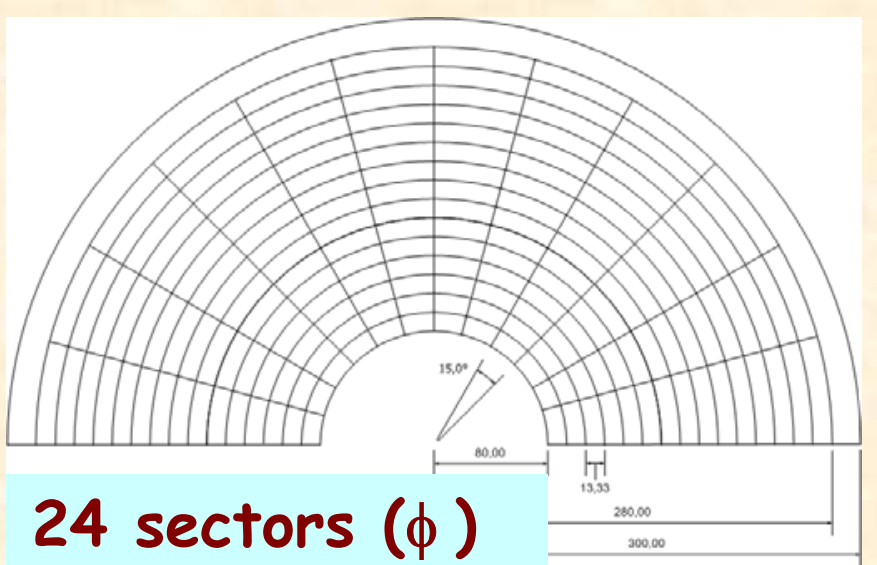

15 cylinders $(r)$

Basic

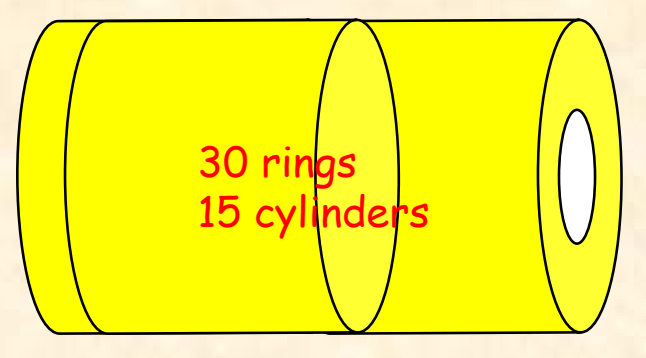

Jinlong Zhang
Maximum Peak Shower

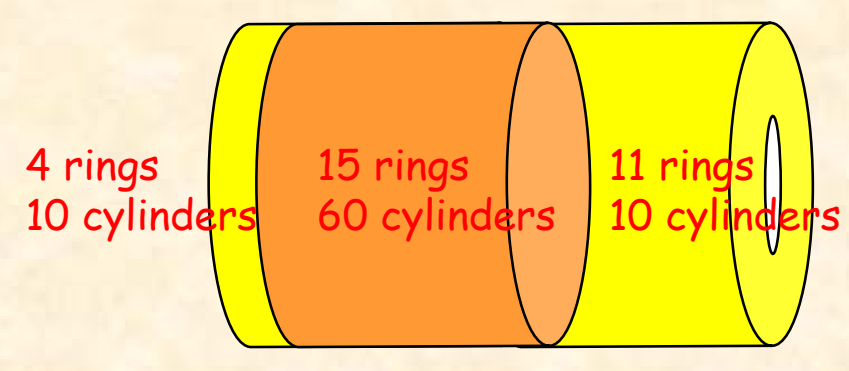




\section{Physics Simulation}

$\star$ Bhabha scattering

BEAM SPREAD, $\sqrt{ } s=500 \mathrm{GeV}$

BHWIDE generator

$\star$ Beam spread

$$
\begin{aligned}
& 0.05 \% \sqrt{S} \\
& 0.5 \% \sqrt{S}
\end{aligned}
$$

$\star$ Beamstrahlung

CIRCE generator
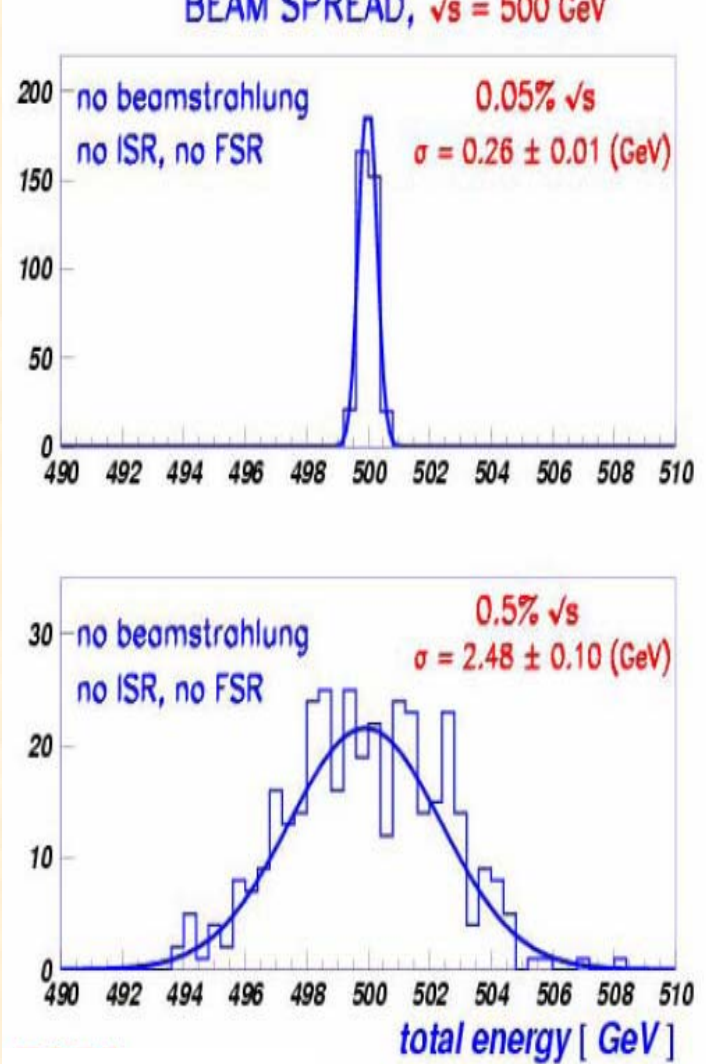

BEAM SPREAD $=0.05 \% \sqrt{\mathrm{S}}$

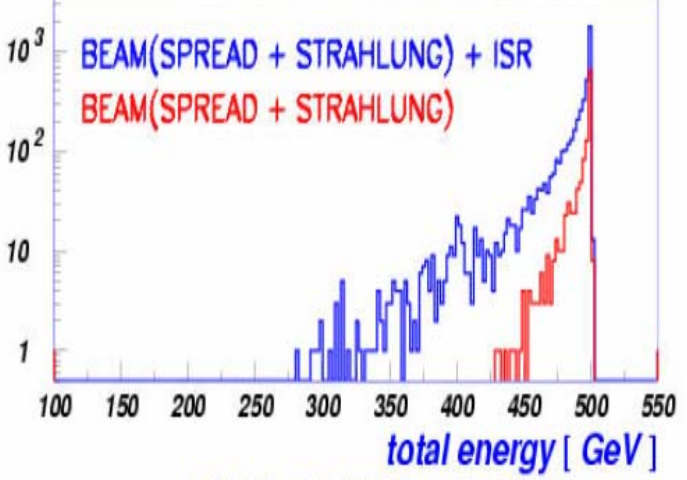

BEAM SPREAD $=0.5 \%$ s

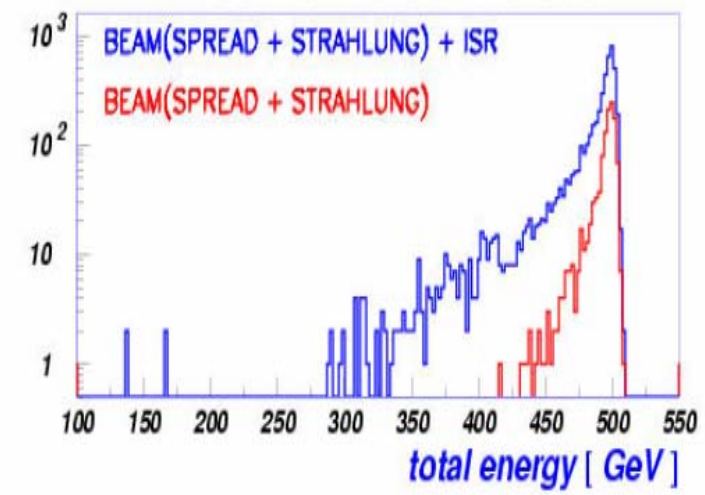




\section{Event Selection}

\section{Symmetry cut}

\section{Geometric Acceptance}
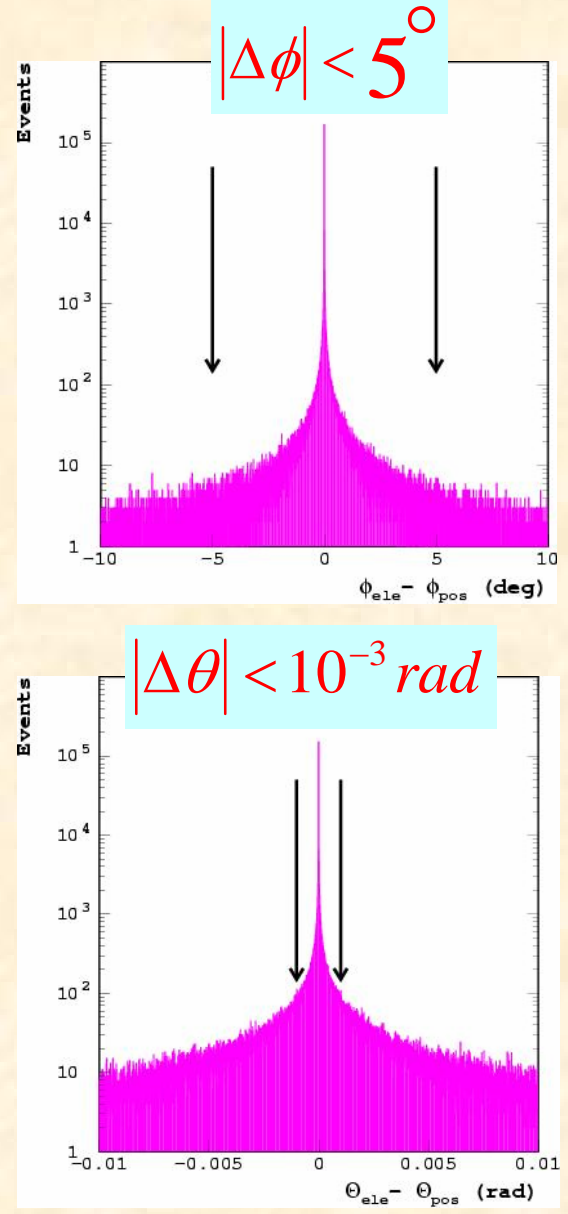
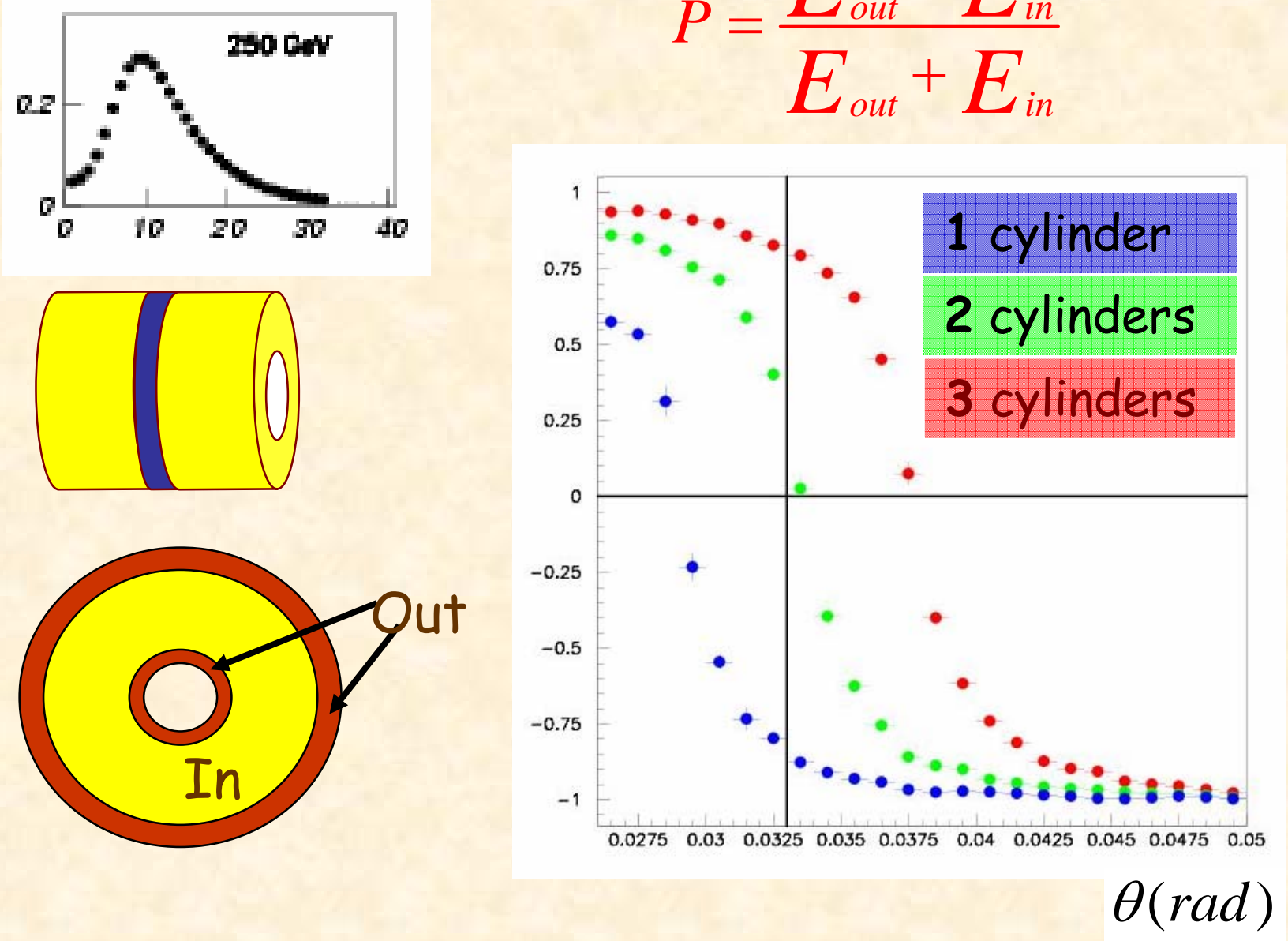

$$
P=\frac{E_{\text {out }}-E_{\text {in }}}{E_{\text {out }}+E_{\text {in }}}
$$

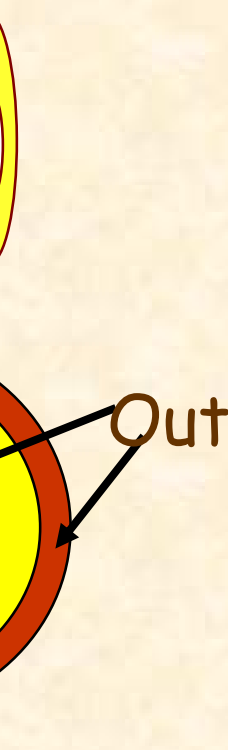




\section{Resolution}

\section{Pad (Basic)}
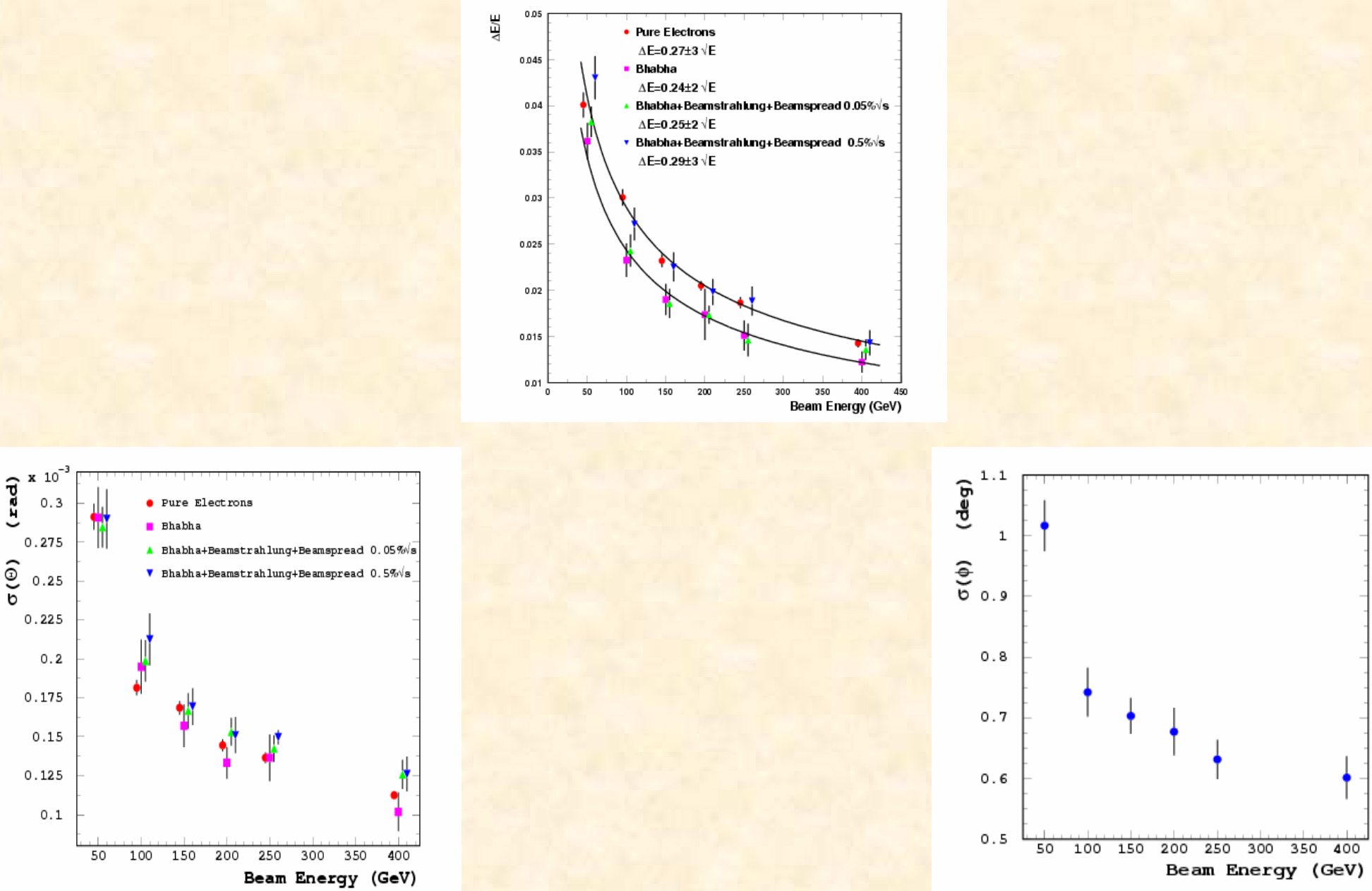


\section{Performance}

\begin{tabular}{|l|c|c|}
\hline \multicolumn{1}{|c|}{ Maximum Peak Shower } \\
\hline Parameter & Pad & Strip \\
\hline Energy resolution & $25 \% \sqrt{ } \mathrm{E}$ & $25 \% \sqrt{ } \mathrm{E}$ \\
\hline$\theta$ resolution (rad) & $3.5 \times 10^{-5}$ & $2.1 \times 10^{-5}$ \\
\hline$\phi$ resolution (rad) & $10^{-2}$ & $10^{-3}$ \\
\hline$\Delta \Theta($ rad) & $\sim 1.4 \times 10^{-6}$ & $\sim 2.1 \times 10^{-7}$ \\
\hline Electronics channels & 25,200 & 8000 \\
\hline
\end{tabular}




\section{Background Suppression}

$\star$ Two-photon process as the main background

$\star e+e-->$ e+e-I+I- $(I=e, \mu, T)$ simulated with WHIZARD
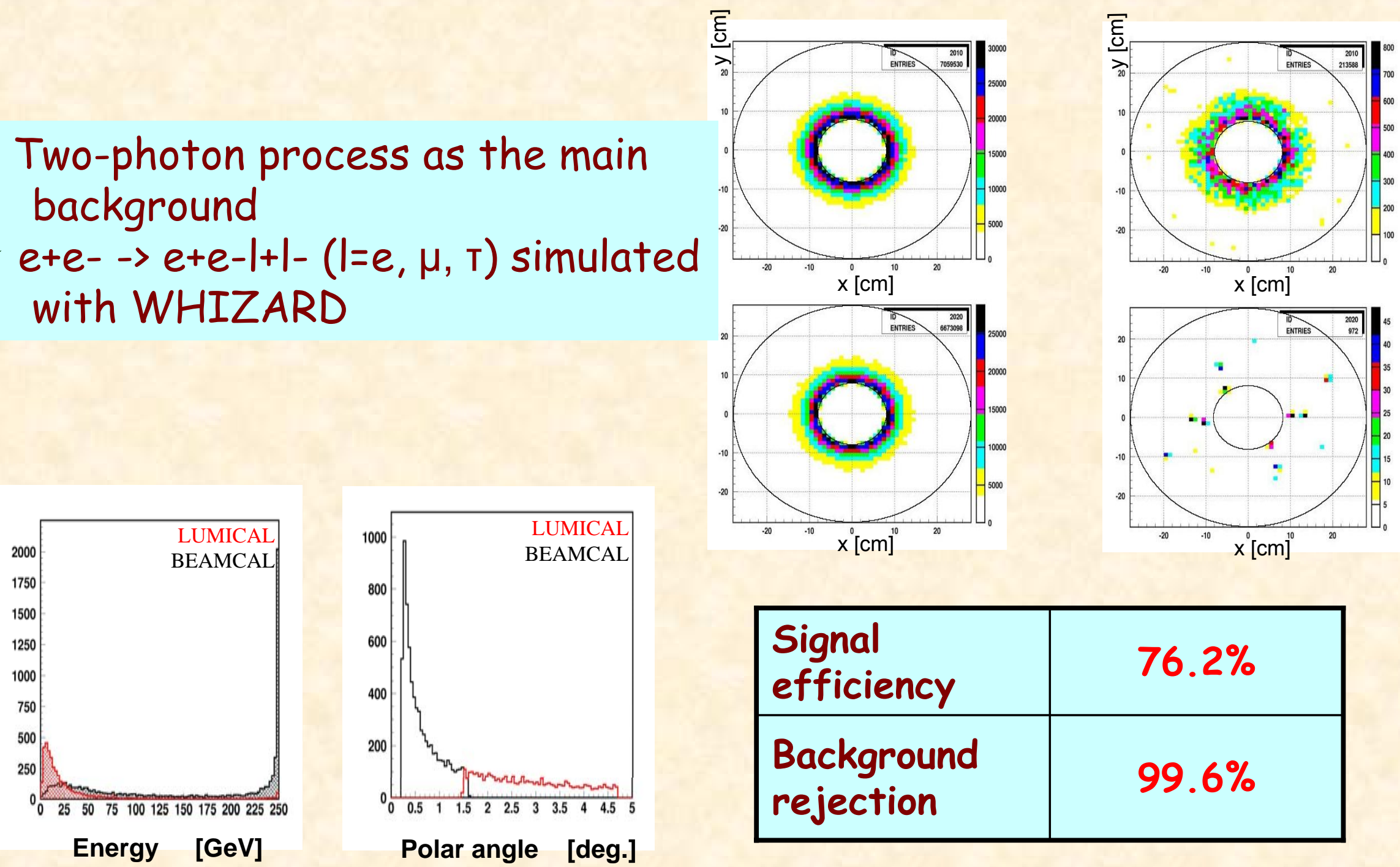

\begin{tabular}{|l|c|}
\hline $\begin{array}{l}\text { Signal } \\
\text { efficiency }\end{array}$ & $76.2 \%$ \\
\hline $\begin{array}{l}\text { Background } \\
\text { rejection }\end{array}$ & $99.6 \%$ \\
\hline
\end{tabular}




\section{Beam Crossing Angle Effect}

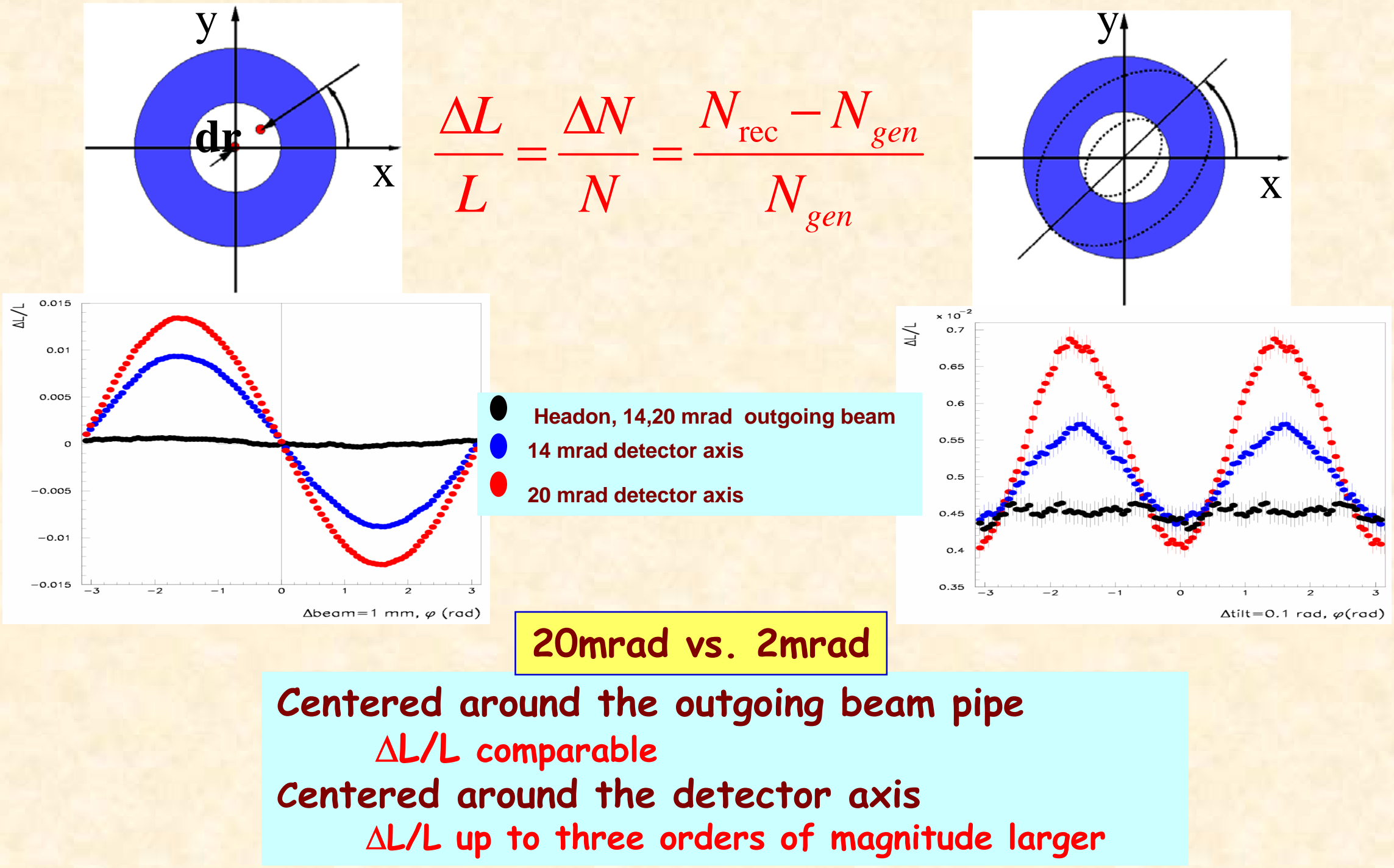




\section{BeamCal}

$\star$ Beam diagnostics

$\star$ Reduction of backscattering to inner subdetectors

$\star$ Detection of high energy electrons and photons
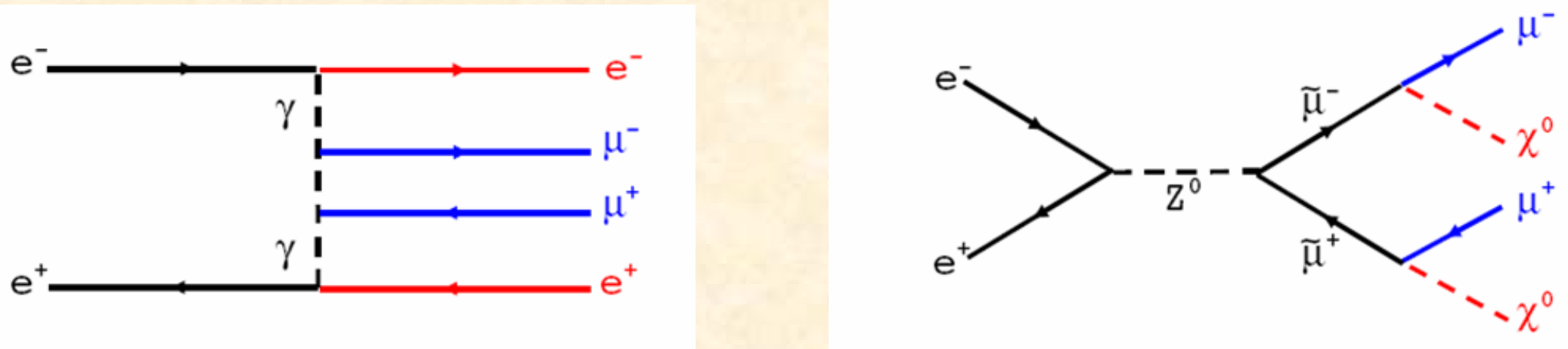

Two photon background

SUSY physics

$\mu^{+} \mu^{-}+$missing energy

$\sigma \sim 10^{6} \mathrm{fb}$

$\mu^{+} \mu^{-}+$missing energy

$\sigma \sim 10^{2} \mathrm{fb}$ 


\section{Geometry}

Sensor: diamond Absorber: tungsten

\begin{tabular}{|l|c|c|c|}
\hline & Head-on & 2 mrad & 20 mrad \\
\hline Absorber thickness $(\mathrm{mm})$ & 3.5 & 3.5 & 3.5 \\
\hline Sensor thickness $(\mathrm{mm})$ & 0.3 & 0.3 & 0.3 \\
\hline X/Y position $(\mathrm{mm})$ & $0 / 0$ & $0 / 0$ & $+36.5 / 0$ \\
\hline$Z$ position $(\mathrm{mm})$ & \pm 3650 & \pm 3650 & \pm 3650 \\
\hline Tilt angle $(\mathrm{mrad})$ & 0 & 0 & 10 \\
\hline $\mathbf{R}_{\text {min }}-\mathbf{R}_{\max }(\mathrm{mm})$ & $15-100$ & $20-100$ & $20-165$ \\
\hline$\theta_{\text {in }}-\theta_{\text {out }}(\mathrm{mrad})$ & $4-28$ & $5-28$ & $5-45$ \\
\hline Number of layers & 30 & 30 & 30 \\
\hline
\end{tabular}




\section{Segmentation}

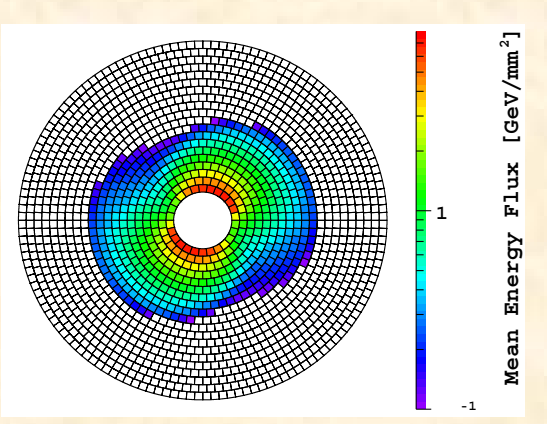

Optimization with

fixed cell size

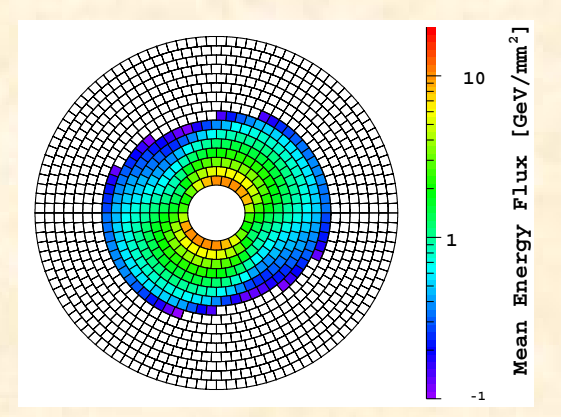

\begin{tabular}{|l|l|c|c|c|c|}
\hline \multirow{4}{*}{$* \boldsymbol{N}^{*} \boldsymbol{N o v}^{v}$} & $4 \mathrm{~mm}$ & $5 \mathrm{~mm}$ & $8 \mathrm{~mm}$ & $10 \mathrm{~mm}$ \\
\cline { 2 - 6 } & Ring & 20 & 16 & 10 & 8 \\
\hline Cell & 1660 & 1072 & 430 & 264 \\
\hline & Channel & 49800 & 32160 & 12900 & 7920 \\
\hline
\end{tabular}

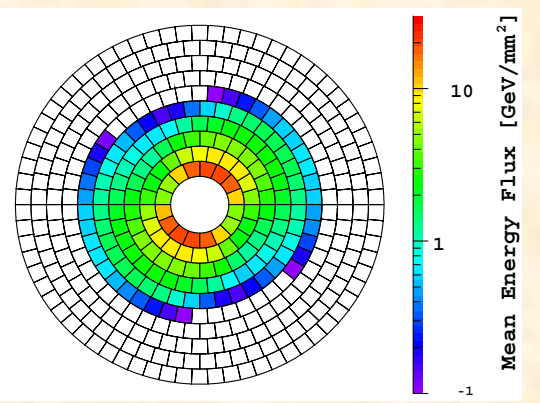

Optimization for electron identification

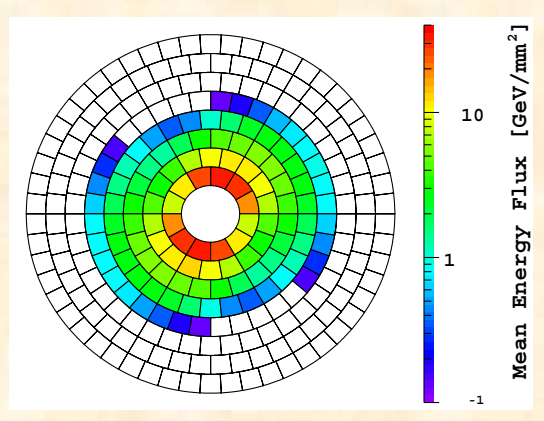




\section{Electron Identification}

$\star$ Use 10 bunches to define $\left\langle E_{b g}\right\rangle$ and $R M S_{E b g}$ for each pad;

$\star$ Subtract $\left\langle E_{b g}\right\rangle$ from $E_{\text {dep }}$ for each pad for a signal event;

$\star$ Keep pads with remaining $E_{\text {dep }}$ larger than $5 \cdot R M S_{E b g}$ :

$\star$ Build clusters:

more than 7 pads in the segment and

more than 4 pads in at least one neighbor segment.
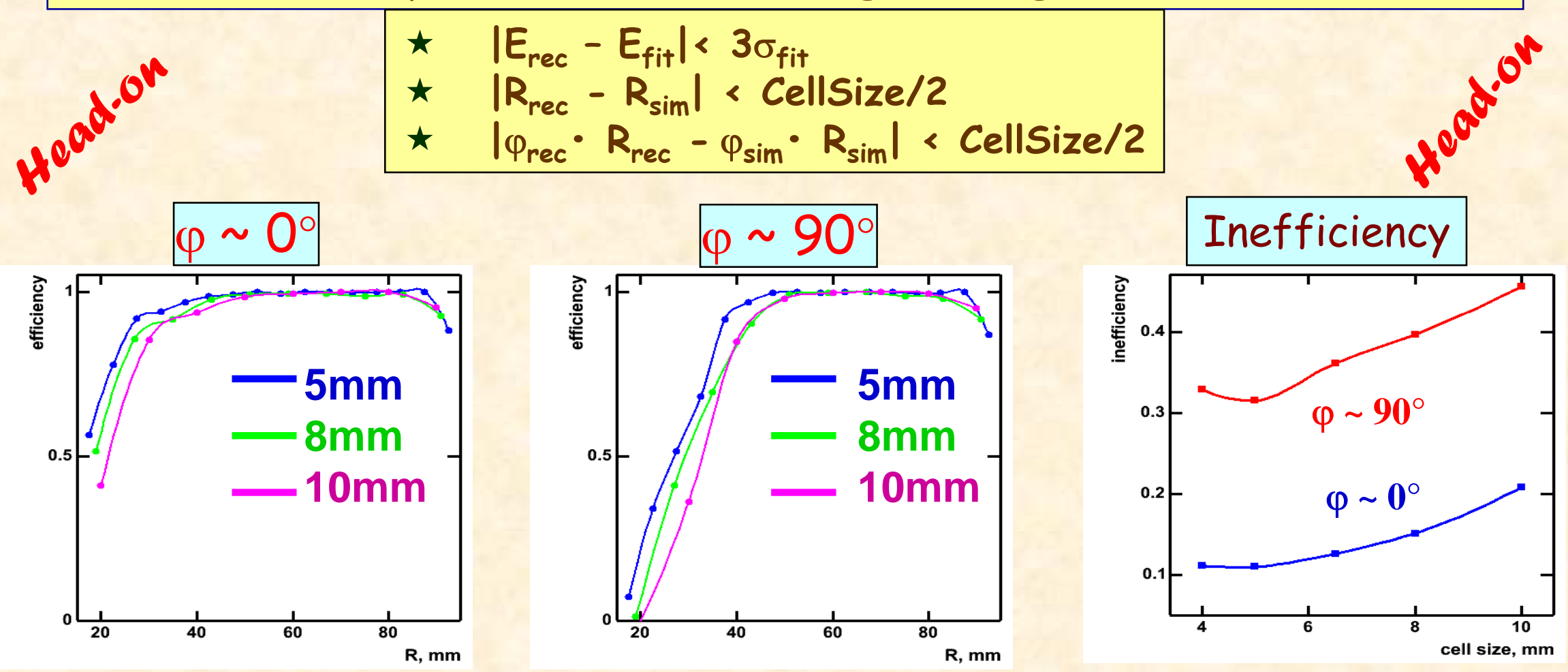


\section{$20 \mathrm{mrad}$ crossing angle \& DID}

$\star$ Detector mounted on the outgoing beam

$\star$ Blind area for the incoming beam

$\star$ Simplified implementation (or B map) of DID

$$
\overrightarrow{\mathbf{B}}=\mathbf{R}(-0.01 \mathrm{mrad})\left(\begin{array}{l}
0 \\
0 \\
4
\end{array}\right)
$$

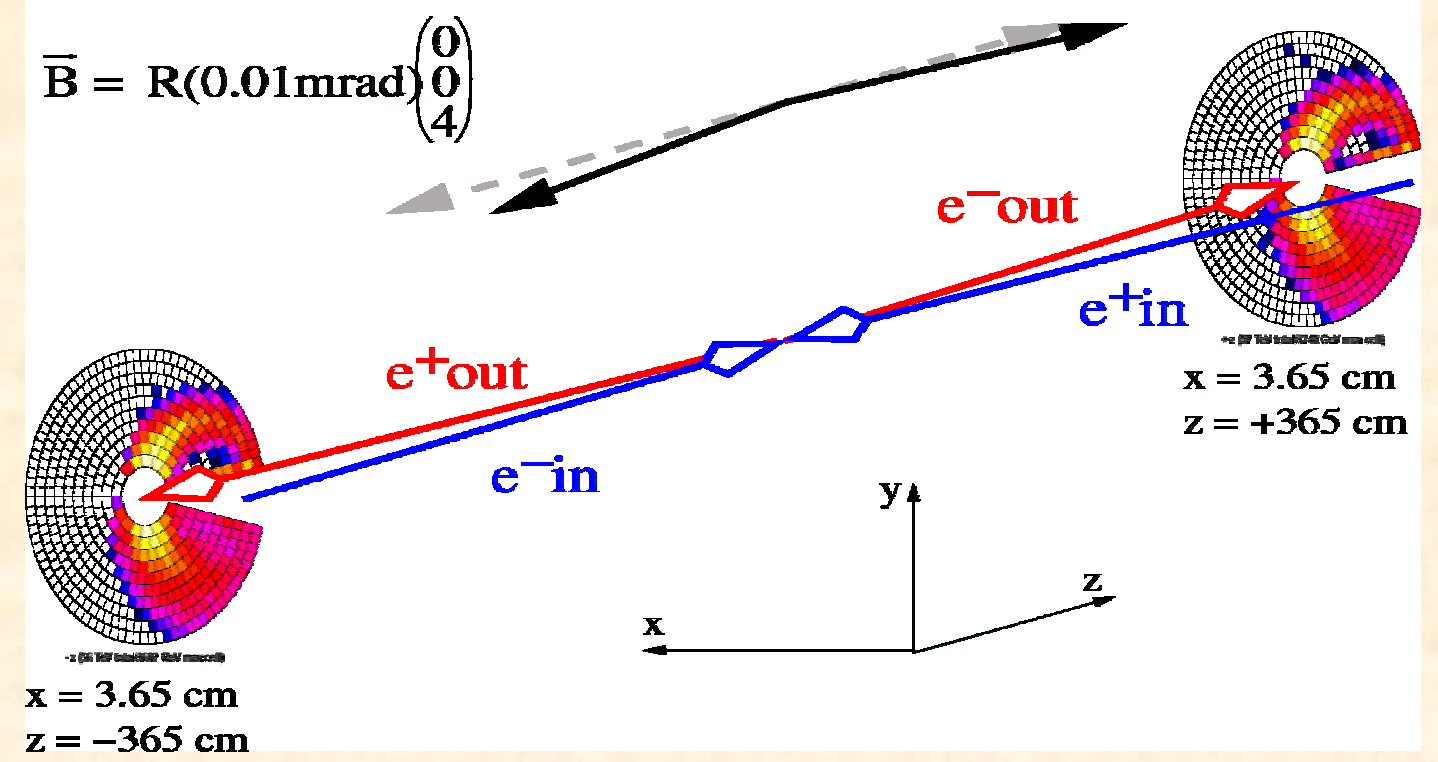




\section{Background}

\section{Head on}

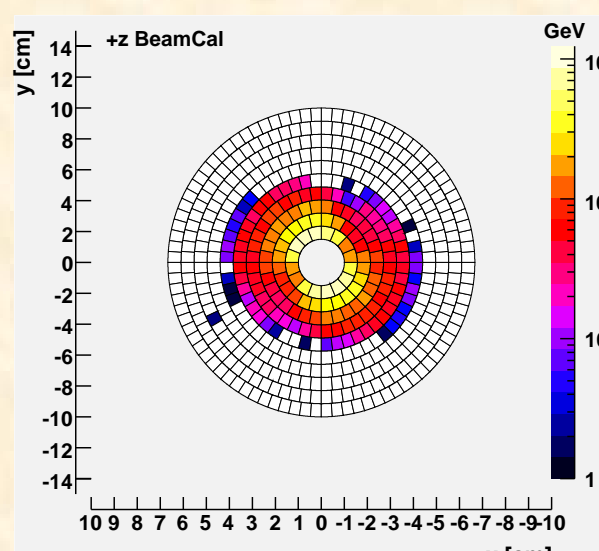

\section{$20 \mathrm{mrad}$ old DID}

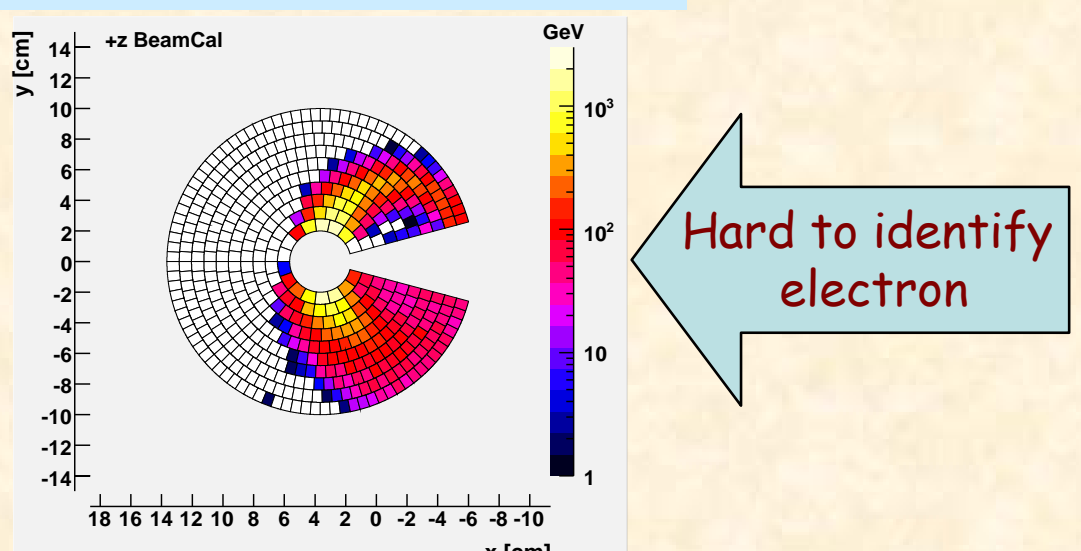

$2 \mathrm{mrad}$

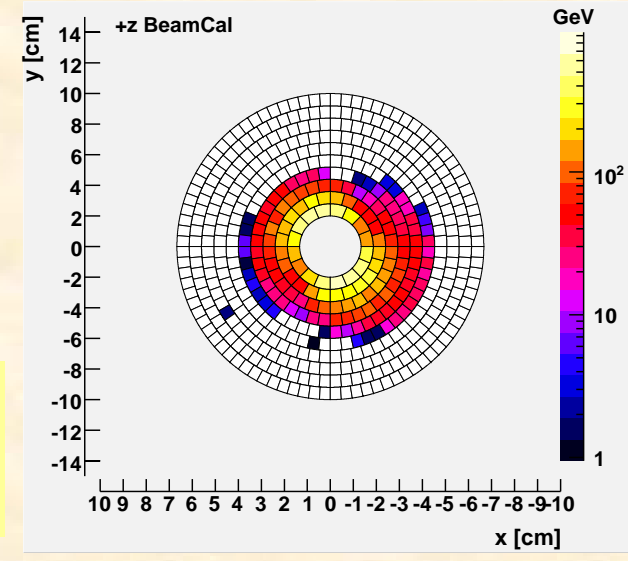

\section{$20 \mathrm{mrad}$ old antiDID}

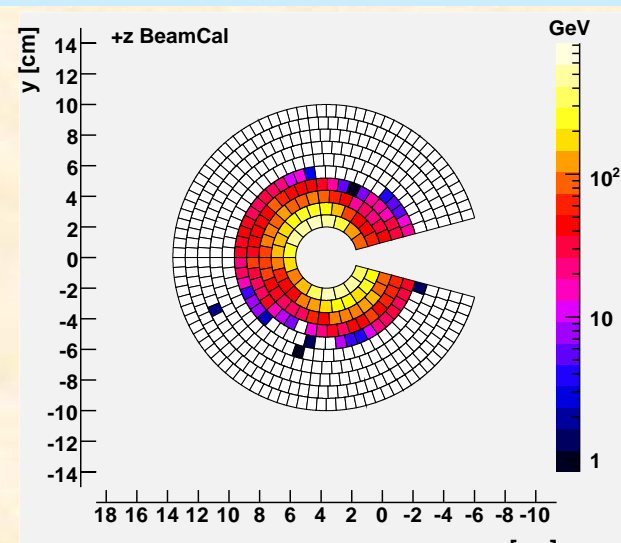




\section{Problem \& Possible Solution}

old
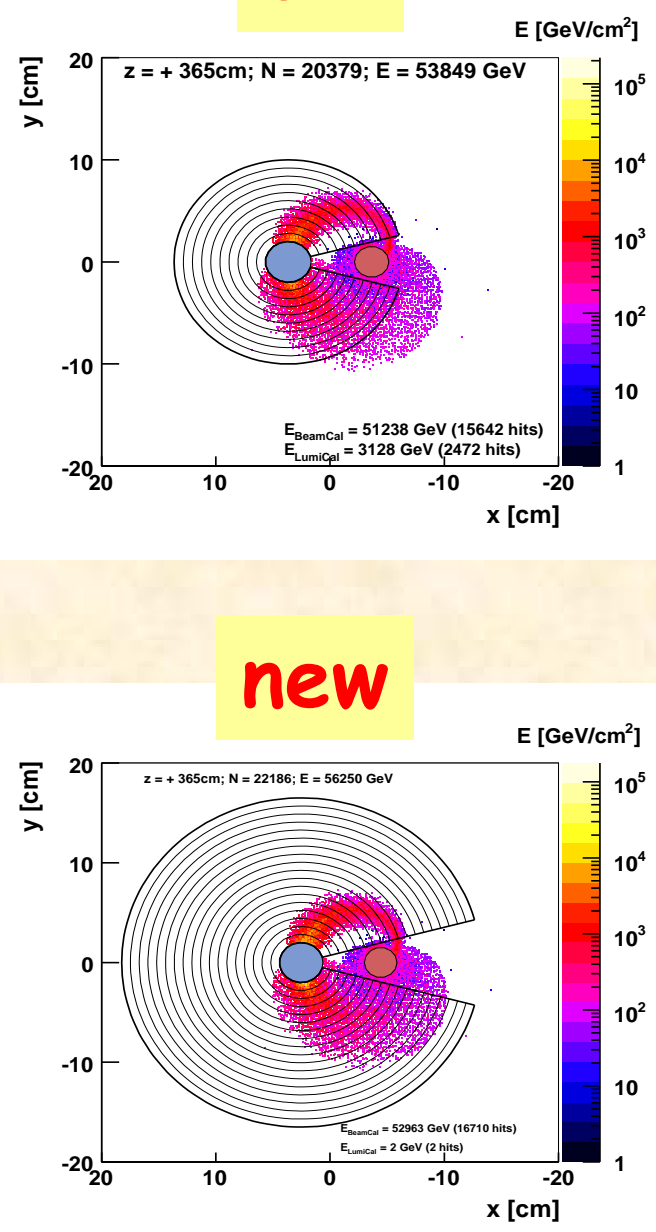

\section{0 mrad new DID}

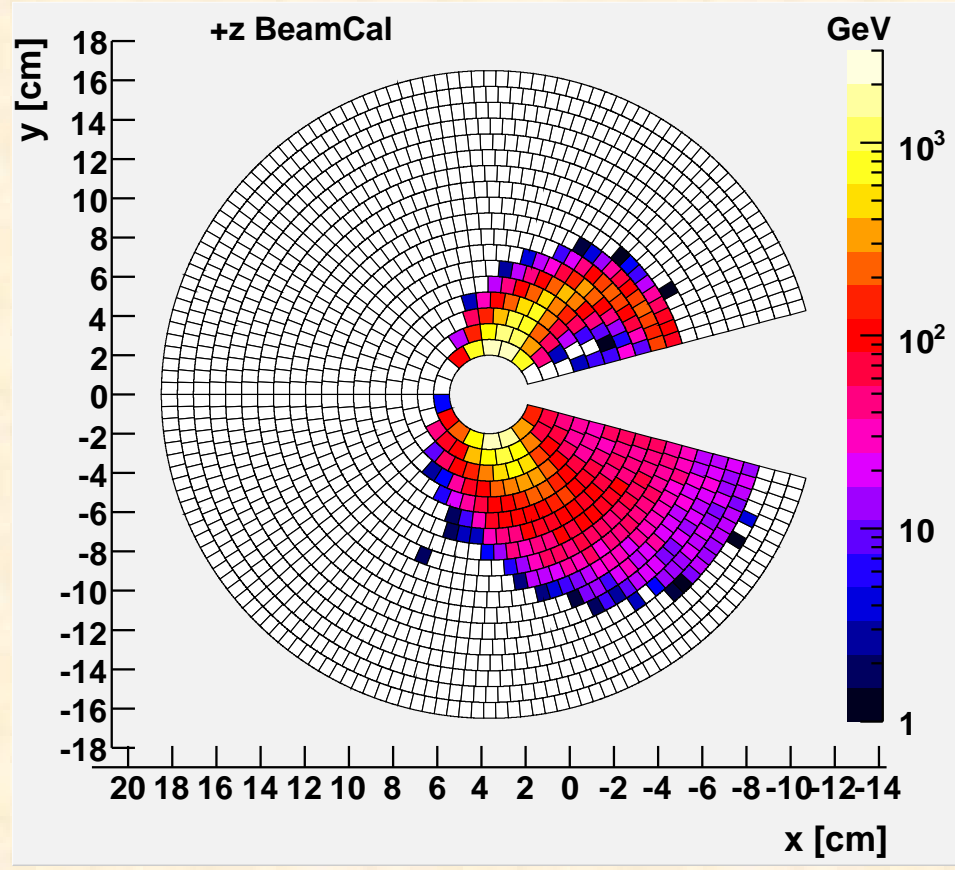




\section{Segmentation with less channels}

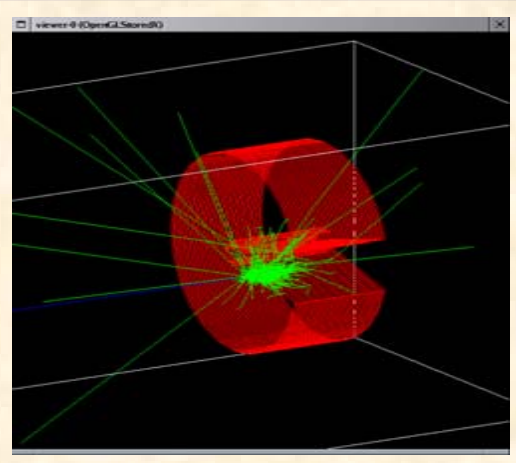

\begin{tabular}{|c|c|c|c|}
\hline & Head-on & $2 \mathrm{mrad}$ & $20 \mathrm{mrad}$ \\
\hline Rings & 10 & 10 & 18 \\
\hline$\Delta R(\mathrm{~mm})$ & 8.5 & 8.0 & 8.06 \\
\hline$N_{\text {seg }}$ in $1^{\text {st }}$ ring & 16 & 16 & 16 \\
\hline$\Delta \mathbf{N}_{\text {seg }}$ per ring & 8 & 8 & 8 \\
\hline $\mathbf{N}_{\text {seg }}$ per layer & 520 & 520 & 1512 \\
\hline Blind area & 0 & 0 & $\pm 15^{\circ}$ \\
\hline
\end{tabular}




\section{Diamond sensor}

Diamond samples (CVD)

- FAP (Freiburg)

- GPI (Moscow)

- Element6 (De Beers)

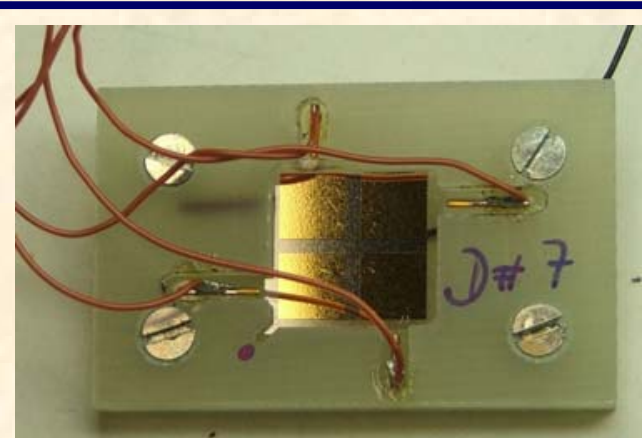

IV behavior

CCD performance

MIP signal

Irradiation

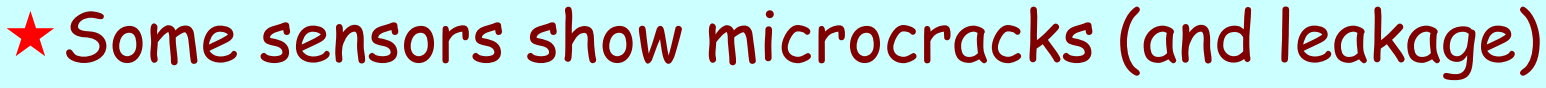

$\star$ CCDs are between 0 and $150 \mu \mathrm{m}$

$\star$ Some sensors are not stable under irradiation

FAP

FAP7_7_p3 CCD vs E-field

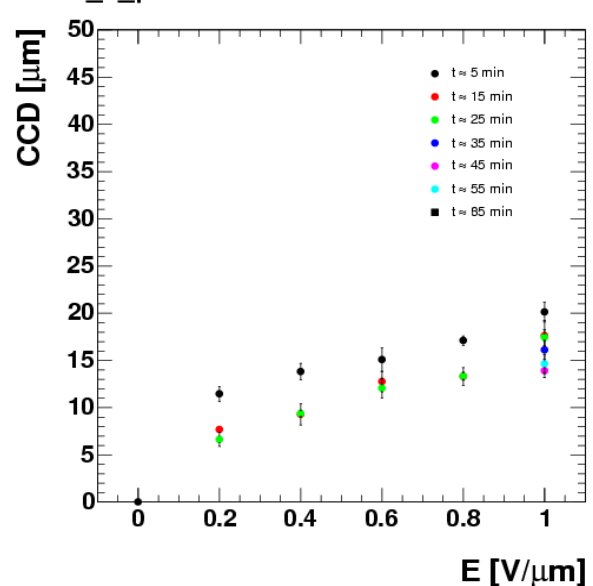

Element 6

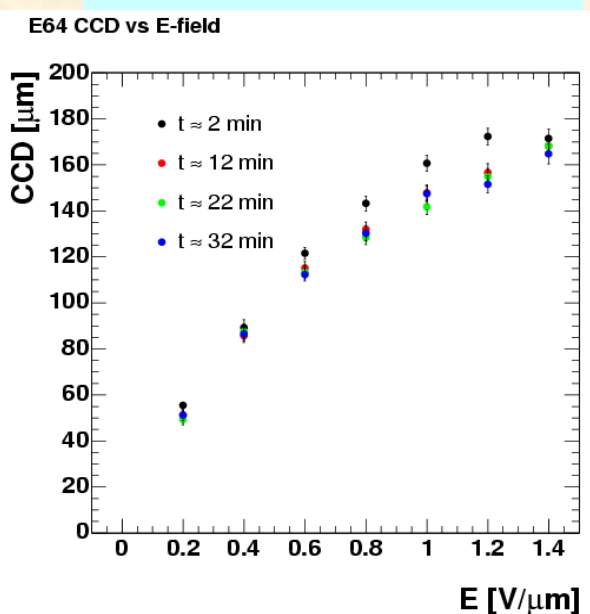




\section{Summary}

$\star$ LumiCal

$>$ Detetor design and performance study with detailed simulation

$>$ Background suppression

$>$ Systematics from geometric distorsion effects

$\times$ Mechaincs and alignment

$x$ Sensor and Readout system

$>$ Achievable $\Delta L / L \approx 10^{-4}$

$\star$ BeamCal

$>$ Detector Design and performance study with different level simulations

x Beam diagnostics to provide many beam parameters

$>$ Different instrumentations

$>$ Sensor testing

$\times$ Electronics design

$\checkmark$ Good progresses by FCAL collaboration. Further optimization is needed. $14 \mathrm{mrad}$ crossing angle? 
